\title{
Wireless Automatic Irrigation System Based On WSN and GSM
}

\author{
Sujit P. Jagtap ${ }^{1}$, Dr. S. D. Shelke ${ }^{2}$ \\ ${ }^{l}$ Department of Electronics and Telecommunication NBN Sinhgad school of Engineering, Pune \\ Pune, India. \\ ${ }^{2}$ Department of Electronics and Telecommunication NBN Sinhgad school of Engineering, Pune \\ Pune, India.
}

\begin{abstract}
This paper describes the Automatic Irrigation based on WSN and Android smart phone for effective water management system. This system focuses on making it possible for the farmers to remotely control and monitor the irrigation system comfortably. However in our research work, we attempt to design the system including the wireless controller via GSM technology. The communication between the sensor (soil moisture) and controller is done with the help of ZigBee module. In addition to that the data which is received from the Irrigation field via GSM is loged on web page using android's operating systems. This software application will control the devices (AC Motors, Valves) wirelessly (GSM). Results from this study found that, the system successfully able to control and monitor irrigation system remotely. The system is seen potentially useful in Irrigation for proper management of water and labor \& cost cutting.
\end{abstract}

Keyword: GSM, ZigBee, Bluetooth, WSN.

\section{Introduction}

Technology development should help people to do their work more easily and make them comfortable. Nowadays wireless technology for an intelligent irrigation system [2], has become important, People are utilizing the merits of embedded system into monitoring and control system for an intelligent irrigation system because of lots of advantages of it. For many, the term 'wireless' is daunting because it brings forth a whole additional terms and acronyms such as WiFi, ZigBee, RFID, WLAN and Bluetooth that are new and intimidating.

India is large agriculture country, and there is a serious shortage of water resources the irrigation water of crop use over $60 \%$ of the total water, and the agriculture water consumption increase year by year. Due to traditional method of irrigation water utilization efficiency is extremely low.

Monitoring parameters of temperature, humidity and moisture in soil is an important means for obtaining high-quality farming. Due to the cost effective nature and deployment flexibility, networks of tiny autonomous sensors capable of wireless communication, have been successfully used in a wide range of applications [1]-[3]. With the help of WSN a very precise knowledge of the evolution of an ecological system can be gained. This precious information helps to understand natural phenomenon like animal tracking, prevent natural disasters such as bush fires and flooding or allow a fine tuning of resources allocation in resourceconstrained areas like irrigation control.

Today, Ethernet network, RF module, zigbee and Bluetooth wireless network are used to transmit data in remote Monitoring System [1]. A -wireless solution it is high costs, hard to be developed and limited signal coverage. While WSN (Wireless Sensor Network) dispenses with the substantial costs of wiring, the Zigbee WSN technologies are most suitable for agriculture applications comparing with Wi-Fi and Bluetooth.

Highly Robustness for the interferences has made ZigBee[3] as a highly versatile and attractive technology among other short range wireless technologies.

Operating over unlicensed, globally available frequency of $2.4 \mathrm{GHz}$.This paper gives a review of remote control and monitoring systems based on existing technologies and a GSM-ZigBee based remote control and monitoring system with automatic irrigation system is proposed here.

The design presented has the advantage of both GSM and ZigBee technology, the sensors and devices are controlled by both by using ZigBee when in a limited range with the appliances and using SMS for remote monitoring and control thereby reducing the usage charges of GSM.

In Addition to that is using android smart phone [6], help to monitor that data on phone easily with the help of SMS reader application. Aim of this research work is to design develop and implement a wireless sensor based communication system[3], to monitor and control the agriculture parameters like temperature and humidity in real time for better management and maintenance of agriculture production and to prevent the sever attack of disease on the crops caused by the climate condition. 
The monitoring system is proposed here basically constitutes sensors for field signal acquisition, microcontroller unit as a front end processing device and ZigBee module to transmit the signal in wireless manner.

\section{Analysis Of Existing System}

There are many existing systems used in Irrigation for remote monitoring and controlling depending upon the requirements. Bluetooth, Wi-Fi, and ZigBee these are the Wireless Sensor Network (WSN) technologies used for wireless communication between the sensors and controllers. For controlling the system microcontroller based or FPGA based system is used depending on requirement.

\section{A. The system implementation using Microcontroller}

Irrigation system based on wireless sensors with GSM-Bluetooth and microcontroller [4], has simpler features designed with the objective of low cost and effective with less power consumption using sensors for remote monitoring and controlling devices which are controlled via SMS using a GSM module. A Bluetooth module is also interfaced with the main microcontroller chip. This Bluetooth module eliminates the usage charges by communicating with the appliances via Bluetooth when the application is in a limited range of few meters.

Highly robustness for the interferences with microcontroller has made Bluetooth as a highly versatile technology among other short range wireless technologies available. Operating over unlicensed, globally available frequency of $2.4 \mathrm{GHz}$, it can link digital devices within a range of $10 \mathrm{~m}$ to $100 \mathrm{~m}$ at the speed of up to $3 \mathrm{Mbps}$ depending on the Bluetooth device class. The advantage of interfacing microcontroller with both GSM and Bluetooth technology and the sensors and devices are controlled by both by using Bluetooth when in a limited range with the appliances and using SMS for remote monitoring and control thereby reducing the usage charges of GSM.

\section{B. The system implementation using FPGA}

An irrigation control system can be developed based on a micro-controller unit (MCU) or an FPGA[5]. An irrigation control system has already been done with 89C52, a kind of MCU. But due to the limitation of the pins of 89C52 (microcontroller), the irrigation controller can only control the irrigation of a single greenhouse or a single piece of field that means it control limited field.

In order to support more requirements, some other peripheral circuits had to be added and more complex solution structure and debugging is resulted in in an irrigation control system based on a MCU.

In comparison, an FPGA has more $\mathrm{I} / \mathrm{O}$ pins and is able to control irrigation of several greenhouses without external interface circuits. It is powerful, flexible and convenient to Complete. all the control logic by means of VHDL or Verilog.

The irrigation control system based on an FPGA is fast in speed, small in size and easy to debug. It is low in cost and presents new approach of the hardware development for irrigation control in the near future.

\section{System Design And Implementation}

Three levels are included in the system: the Android smart phone the controller and the action unit. Simple GSM modules are available in the PC control platform and the controller. Orders can be sent from the PC control platform or cell phone to the controller and the information such as temperature, soil moisture and air humidity sampled by the controller can also be sent to the PC platform or cell phone by GSM message.

Emitter and receiver of short-wave radio are embedded in the controller and the action unit respectively. Radio communication ZigBee) works between the controller and the action units.

\section{Controller and Sensors}

The LPC2131/32/34/36/38 microcontrollers are based on a 16/32-bit ARM7TDMI-S CPU with realtime emulation and embedded trace support, that combine the microcontroller with $32 \mathrm{kB}, 64 \mathrm{kB}, 128 \mathrm{kB}, 256$ $\mathrm{kB}$ and $512 \mathrm{kB}$ of embedded high-speed flash memory. Due to their tiny size and low power consumption, these microcontrollers are ideal for applications where miniaturization is a key requirement, such as access control and point-of-sale. With a wide range of serial communications interfaces and on-chip SRAM options of $8 \mathrm{kB}$, $16 \mathrm{kB}$, and $32 \mathrm{kB}$, they are very well suited for communication gateways and protocol converters, soft modems, voice recognition and low-end imaging, providing both large buffer size and high processing power. Various 32bit timers, single or dual 10-bit 8-channel ADC(s), 10-bit DAC, PWM channels and 47 GPIO lines with up to nine edge or level sensitive external interrupt pins make these microcontrollers particularly suitable for industrial control and medical systems.

PIC 16F877A is adopted to be the microprocessor The characteristic of this chip is listed as following: $3.3 \mathrm{~V}$ power supply and 5 power saving modes, 256Kbyte flash EEPROM, 368bytes SRAM, 8 channel 10 bits 
AD converters, 1 programmable USARTs and two 8-bit and one 16-bit timers. SIM300 GSM module is used in this application.GSM module is connected to PIC 16 F877 by RS-232 interface in TTL.

The LM35 series are precision integrated-circuit temperature sensors, with an output voltage linearly proportional to the Centigrade temperature. Thus theLM35 has an advantage over linear temperature sensors calibrated in ${ }^{\circ}$ Kelvin, as the user is not required to subtract a large constant voltage from the output to obtain convenient Centigrade scaling. The LM35 does not require any external calibration or trimming to provide typical accuracies of $\pm 1 / 4{ }^{\circ} \mathrm{C}$ at room temperature and $\pm 3 / 4^{\circ} \mathrm{C}$ over a full $-55^{\circ} \mathrm{C}$ to $+150^{\circ} \mathrm{C}$ temperature range. Low cost is assured by trimming and calibration at the wafer level.

The low output impedance, linear output, and precise inherent calibration of the LM35 make interfacing to readout or control circuitry especially easy. The device is used with single power supplies, or with plus and minus supplies. As the LM35 draws only $60 \mu \mathrm{A}$ from the supply, it has very low self-heating of less than $0.1{ }^{\circ} \mathrm{C}$ in still air. The $\mathrm{LM} 35$ is rated to operate over a $-55^{\circ} \mathrm{C}$ to $+150^{\circ} \mathrm{C}$ temperature range, while the $\mathrm{LM} 35 \mathrm{C}$ is rated for $\mathrm{a}-40^{\circ} \mathrm{C}$ to $+110^{\circ} \mathrm{C}$ range $\left(-10^{\circ}\right.$ with improved accuracy). The soil moisture sensor's output signal is voltage between 0 to $1.12 \mathrm{~V}$ or current between 0 to $20 \mathrm{~mA}$.Xbee-pro is ZigBee module is used.

\section{Android Smart Phone}

Android smart phone is used here to remotely control the motor and valves by sending command as SMS via mobile while will receive by the GSM module interface with controller. Also the information sent back by GSM module such as temperature, soil moisture is uploaded on web using android phone.

\section{E. Action Unit}

The action unit consist of motors and valves which remotely operated by the user using GSM mobile. according to the user command action unit is controlled by the microcontroller which is interfaced with the controller.

\section{Proposed System}

The system block diagram is shown in Fig.1, the hardware consists of microcontroller, temperature sensor, soil moisture sensor, water level sensor, GSM module, ZigBee module and android phone. GSM module which is interface with microcontroller receives the command/SMS from user GSM mobile. The SMS is decoded by controller and according to that take action. Before turn on the motor the water level of reservoir is check by the controller and this data is sent back to user as SMS.

It also send data about temperature, humidity and status of motor. The data regarding temperature and humidity is uploaded on web page with the help of android mobile so that anyone can access that info. The block diagram of the proposed GSM-ZigBee based system is given in fig. 1. In this system both GSM and ZigBee modules are interfaced with the main controller chip. GSM is used for remotely monitoring and controlling the devices via a mobile phone by sending and receiving SMS via GSM network.

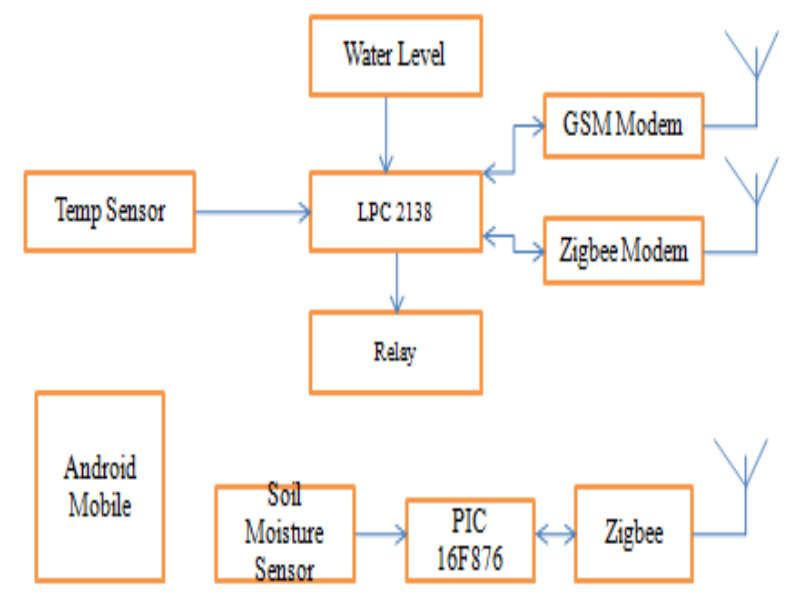

Fig 1. System block diagram.

The motor pumps and valves are controlled automatically using sensor and the other appliances are controlled by GSM network via SMS. The system informs user about any abnormal conditions like low water detection and temperature rise via SMS from the GSM Module to the user's mobile and actions are taken accordingly by the user. This leads to efficient utilization of power. Even using ZigBee module, by SMS, pump and valves could be automatically ON or OFF. 


\section{System Flowchart}

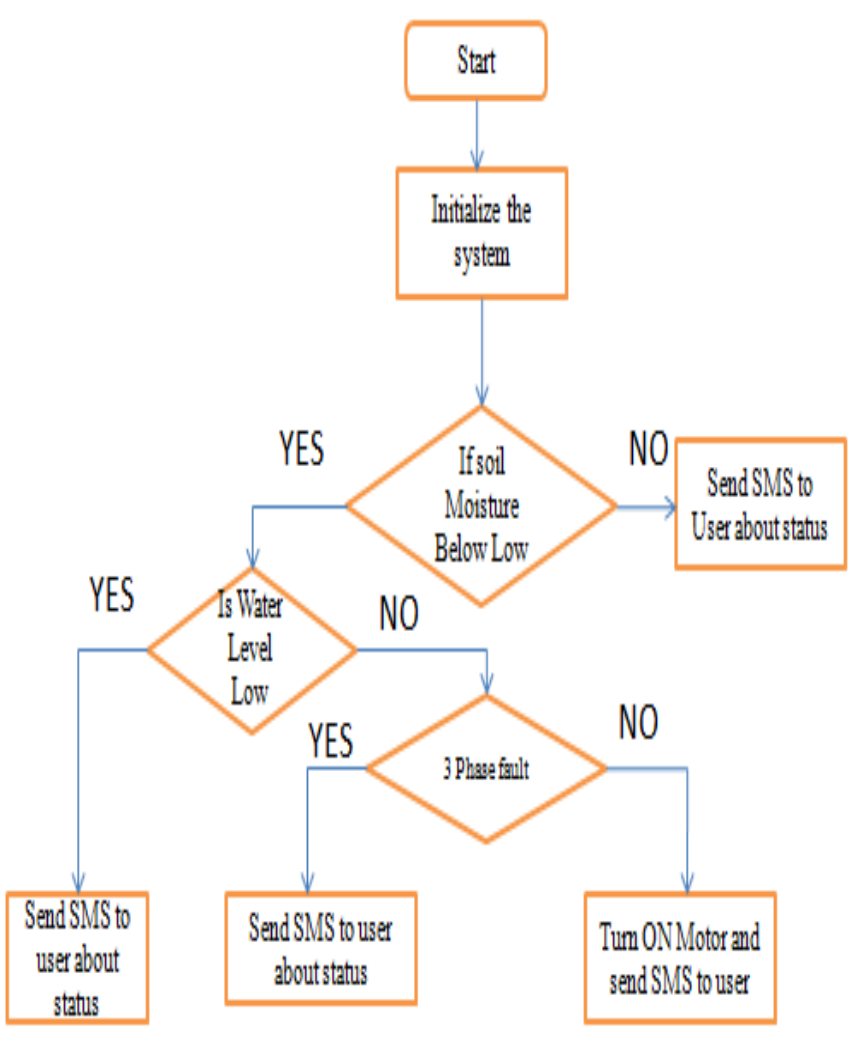

Fig 2. System flowchart.

The Algorithm shown in Fig. 2.consist of following steps

1. Initialize the system first.

2. Check the soil moisture level if it is below low then check the another two conditions that is about water level and phase fault

3. If these all 3 conditions are satisfied then only Motor will On then the information about the status about the these parameters will send to user as SMS via GSM modem.

4. After some time again check all 3 conditions, if soil moisture is above low then motor will OFF.

5. Stop.

\section{F. Hardware result}

\section{Results}

The actual hardware implementation of the system is shown in Fig. 3 which shows interfacing of GSM module, LM 35 temperature sensor, water level sensor with the LPC2138 microcontroller. The SMS send by the GSM module is received on mobile which is as in Fig.3. The status about temperature, phase fault, water level is also shown on LCD which is interfaced with LPC2138 microcontroller.

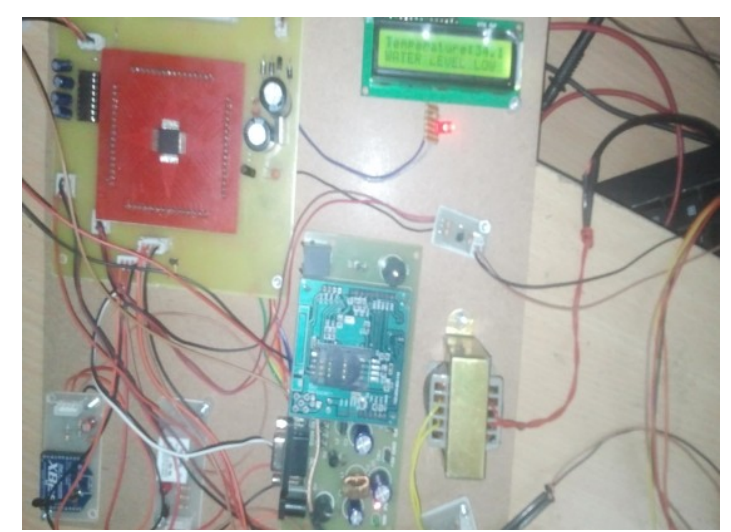

Fig 3. System implementation result 


\section{G. Simulation result}

The simulation result shown in fig.4 shows how the SMS is send to number which is predefined in code and the AT commands used for sending SMS from GSM module. this simulation is done using Docklight simulation software. The message contain the status about phase fault, water level sensor and soil moisture.

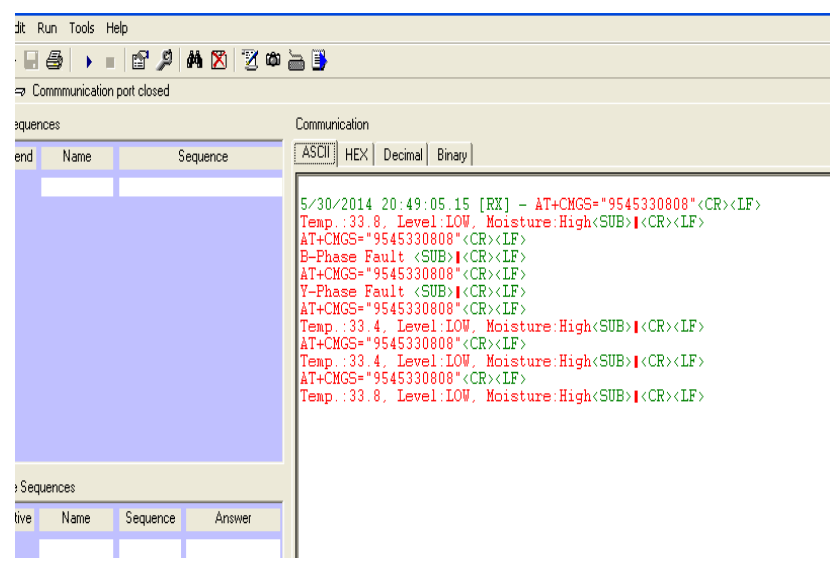

Fig 4. System block diagram.

\section{Conclusion}

In traditional irrigation method the water utilization efficiency is extremely low. This paper proposed wireless water saving system based on GSM and ZigBee network which is used to remotely monitor and control the irrigation system, which save the water and labor cost.

\section{References}

[1] Genghuang Yang, Yuliang Liu, Li Zhao, Shigang Cui, “Automatic Irrigation System Based on Wireless Network", $8^{\text {th }}$ IEEE International Conference on Control and Automation,pp.2120-2125, June 9-11, 2010.

[2] Yunseop Kim, Robert G.Evans, "Remote Sensing and Control of an Irrigation System Using Distributed Wireless Sensor Network", IEEE Transaction on Instrumentation and Measurement, Vol.57, pp.1379-1387, No.7, July 2008.

[3] Akshay C, Nitin Karnwal, Ezhilarasi D, "Wireless Sensing and control for Precision Green House Management", $6{ }^{\text {th }}$ International Conference on Sensing Technology(ICST), pp.52-56,2012.

[4] Zhiyong Lai, Yongli Dai,"An Irrigation System Based on An FPGA", $2^{\text {nd }}$ IEEE International Conference on Instrumentation \& Measurement, pp.159-163, 2012.

[5] Won-Jae Yi, Weidi Jia ," Mobile Sensor Data Collector Using Android Smartphone”, 55th IEEE International Midwest symposium on circuits and systems (MWSCAS), pp.956-959, 2012..

[6] L.L.Pfitscher,"Automatic Control Of Irrigation System Aiming at High Energy Efficiency in Rice Crops", 8 th $I E E E$ International Caribbean Conference on Devices,Circuits and Sysems(ICCDCS) pp.1-4,2012. 